\title{
Geometry Optimization and Stability of Hydrated Trialanine: FMO/EFP Study
}

\author{
M. RAMEGOWDA
}

Research Centre, Post-Graduate Department of Physics, Government College (Autonomous), Mandya-571401, India

hmrgowda@yahoo.com

Received 8 November 2012 / Accepted 15 December 2012

\begin{abstract}
The fragment molecular orbital (FMO) method interfaced with effective fragment potentials (EFP), denoted by FMO/EFP, was developed and applied to polypeptides solvated in water. The structures of neutral and zwitterionic trialanine immersed in water layers of 3.0, 3.5, 4.0, 4.5 and $5.0 \AA$ were investigated by performing FMO/EFP geometry optimizations at the RHF/cc-pVDZ level of theory. Using the optimized geometries, the relative stability of the hydrated zwitterionic and neutral structures is discussed structurally and in terms of energetics at the second-order Møller-Plesset theory (MP2)/cc-pVDZ level.
\end{abstract}

Keywords: FMO, EFP, RHF, MP2, Trialanine, cc-pVDZ

\section{Introduction}

The fragment molecular orbital (FMO) method $^{1-4}$ which has a nearly analytic energy gradient $^{5-7}$ and closely reproduces energies obtained from the corresponding ab initio method at the same level of theory. The FMO method has been used to do molecular dynamics (MD) ${ }^{8-11}$ calculations. A fully analytic gradient will facilitate MD simulations at high levels of theory ${ }^{12}$.

Since most biological processes occur in solution, solvent effects must also be considered. The biologically relevant form of amino acids is the zwitterionic form and are essentially always in this form at neutral $\mathrm{pH}^{13,14}$. Zwitterionic species of amino acids have both a negatively charged carboxylate group $\left(\mathrm{COO}^{-}\right)$and a positively charged ammonium group $\left(\mathrm{NH}_{3}{ }^{+}\right)$. They are the dominant form in aqueous solution over a wide range of $\mathrm{pH}$. In contrast, in the gas phase, where interactions with environment are not present, amino acids are mostly in their neutral form ${ }^{15-18}$. The fact that the hydrated amino acids are zwitterions, implies that interactions with the water molecules are a key determinant of the stable zwitterionic structure. Alanine is the smallest chiral $R$-amino acid, with a nonreactive methyl group $\left(-\mathrm{CH}_{3}\right)$ as the side chain. It is one of the aliphatic amino acids that have the important property of not interacting favorably with water. It is considered to be hydrophobic and is normally placed on the hydrophobicity scale after glycine, leucine, isoleucine and valine. ${ }^{19,20}$ 
To study the effect of hydration, an explicit solvent treatment can be performed directly with the polarizable continuum model $(\mathrm{PCM})^{21,22}$. The effective fragment potential (EFP) method $^{23,24}$ is a model potential derived from first principles quantum chemistry. In the combined FMO/EFP method ${ }^{25}$, solvent molecules are treated by EFP and the solute molecule by FMO. Intensive studies have been performed on solvated alanine by many researchers ${ }^{26,27}$, Jensen and Gordon ${ }^{28}$ reported that a zwitterionic glycine molecule with two water molecules is a local minimum, based on correlated $a b$ initio calculations with polarization basis functions. However, with two water molecules, the neutral isomer is still lower in energy. Aikens and Gordon ${ }^{29}$ discussed the importance of bulk water for the stability of zwitterionic glycine by applying the QM method for the important water molecules that are directly interacting with glycine and PCM for bulk water.

Within the scope of this work, the attempt is to determine the structure and properties of the hydrated trialanine and describe the neutral and zwitterion minimum energy structures and their stability in a polar water environment. The effect of solvation has to be included in simulations to achieve physical meaningful results ${ }^{30}$, especially for vibrational spectra ${ }^{31-36}$. Nevertheless, the trialanine zwitterion in aqueous solution still remains poorly studied. Yuguang et al. studied the conformational dynamics of trialanine in water ${ }^{37}$. An analysis of the conformational dynamics of an alanine tripeptide analogue in the gas phase ${ }^{38}$, CarParrinello molecular dynamics study of the effect of protonation in a hydrated glycine molecule ${ }^{39}$ and stability of tetraglycine ${ }^{12}$ have been reported.

In this work, minimum energy structure and stability of neutral and zwitterionic solvated trialanine molecule is performed. The use of the FMO/EFP based QM/MM method allows one to consider explicitly the study of interaction energy between solvent and peptide molecules.

\section{Computational details}

Trialanine molecule in both neutral and zwitterionic form were modeled using molecular modeling software Avogadro ${ }^{40}$. The molecular modeling software VEGA $^{41}$ was used to construct water layers of 3.0, 3.5, 4.0, 4.5 and $5.0 \AA$ from trialanine, defined as the closest atom-atom distance from the solute to the solvent. The FMO-RHF/EFP geometry optimization calculations were then carried out at the RHF/cc-pVDZ level of theory ${ }^{42}$ implemented in the GAMESS-US software suit ${ }^{43,44}$. To obtain the energies of the zwitterionic form of hydrated trialanine relative to those of the neutral form, the numbers of water molecules must be the same for each water layer but a slightly different number is generated by VEGA. To avoid this problem, a few water molecules were removed; for example, at the $5.0 \AA$ water layer consisting of 70 and 79 water molecules for the neutral and zwitterion, respectively, nine water molecules in the zwitterionic system, which are far away from the solute, were removed. The energies of hydrated trialanine, free solute and solvent (EFP) for both neutral and zwitterionic forms were carried out at the MP2/cc-pVDZ level of theory.

To study the relative stabilities of hydrated zwitterionic trialanine systems by comparing their energies with those of the hydrated neutral systems, the relative energy $\Delta E^{\text {tot }}$ is estimated by subtracting the total energy $E^{\text {neu }}$ of the hydrated neutral system from that of the corresponding hydrated zwitterionic system $E^{z w i t}$, i.e.,

$$
E^{\text {tot }}=E^{\text {zwit }}-E^{\text {neu }}
$$

The optimized geometry for solvated trialanine is used to compute the energy of the free solute (solu), $E^{\text {solu,zwit }}$ and $E^{\text {solu, neu }}$, by removing solvent molecules from the system. Similarly, removing the solute allows one to compute the energy of the free solvent (solv) $E^{\text {solv,zwit }}$ and $E^{\text {solv, neu }}$. Then, the solvent-solute interaction energies are 


$$
\begin{aligned}
& E^{\text {solu-solv,zwit }}=E^{\text {zwit }}-\left(E^{\text {solu,zwit }}+E^{\text {solv,zwit }}\right) \\
& E^{\text {solu-solv,neu }}=E^{\text {neu }}-\left(E^{\text {solu, neu }}+E^{\text {solv, neu }}\right)
\end{aligned}
$$

and the relative energy can be decomposed as

$$
\Delta E^{\text {tot }}=\Delta E^{\text {solu }}+\Delta E^{\text {solv }}+\Delta E^{\text {solu-solv }}
$$

Where $\Delta E^{\text {solu }}=\Delta E^{\text {solu,zwit }}-\Delta E^{\text {solu,neu }}$ describes the relative stability of two forms of trialanine without solvent, $\Delta E^{\text {solv }}=\Delta E^{\text {solv, zwit }}-\Delta E^{\text {solv, neu }}$ describes the stability of solvent in the two hydrated forms of trialanine and $\Delta E^{\text {solu-solv }}=\Delta E^{\text {solu-solv, zwit }}-\Delta E^{\text {solu-solv, neu }}$ is the relative value of the solute-solvent interactions in the two forms of trialanine.

\section{Results and Discussion}

Figure 1 and Figure 2 depict the optimized solute structures of the neutral and zwitterionic trialanine in gas phase and in solvent phase, respectively. Figure 3 displays the hydrated neutral and zwitterionic trialanine in the water layer of thickness 5.0 A.

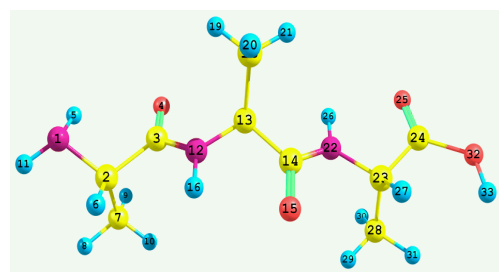

(a)

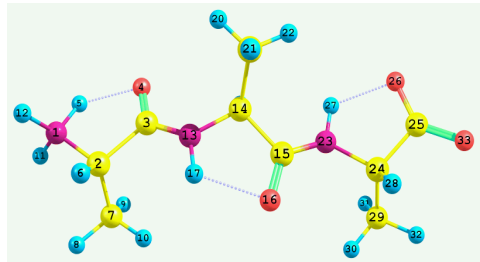

(b)

Figure 1. Optimized structures of trialanine (a) neutral and (b) zwitterionic form in gas phase. (Red-O, Magenta-N, Yellow-C and Cyan-H)

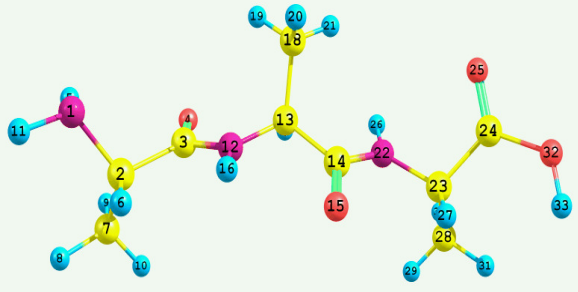

(a)

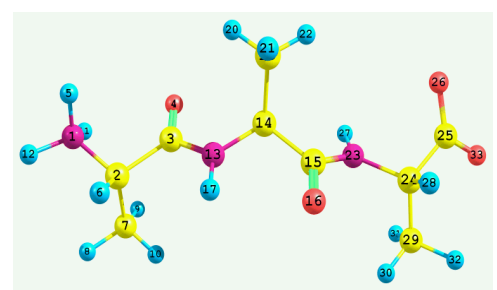

(b)

Figure 2. Optimized solute structures of trialanine (a) neutral and (b) zwitterionic form in solvent (water) phase. (Red-O, Magenta-N, Yellow-C and Cyan-H)

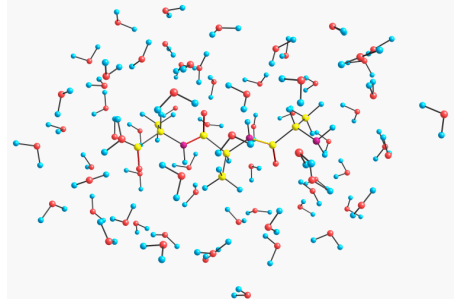

(a)

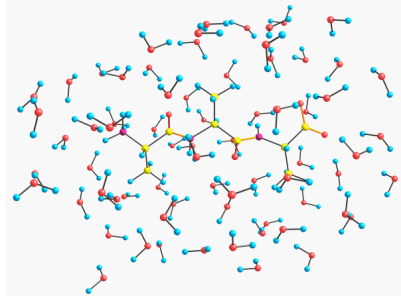

(b)

Figure 3. Optimized structures of hydrated (water layer of $5.0 \AA$ ) trialanine (a) neutral and (b) zwitterionic form. (Red-O, Magenta-N, Yellow-C and Cyan-H) 
Table 1 presents the total relative energy with the incremental thickness of EFP water layers. The negative sign in the relative energies $\Delta E^{\text {tot }}$ means that the zwitterionic system is more stable, as may be seen in Eqs. (2) and (3). The relative energy contributions within the solute molecules, $\Delta E^{\text {solu }}$ in the second column (the standalone solute energies) do not change very much with the increase in the number of water layers. The zwitterionic system always gains stability relative to the neutral system. The solvent internal energies, $\Delta E^{\text {solv }}$ (third column in Table 1), increase with the number of water layers but not monotonically. This implies that the hydrogen bond networks of the water clusters under the influence of neutral trialanine are always more strongly bound. In contrast, the fourth column of Table 1 (the solute-solvent interaction energy: $\Delta E^{\text {solu-solv }}$ ) shows that the solute-solvent relative energies are more negative (more strongly bound) for the zwitterionic systems than for the neutral systems, with strong interactions between the charged groups within the zwitterion and weaker hydrogen bond networks within the water cluster (given by $\Delta E^{\text {solv }}$ ). The values of $\Delta E^{\text {solv }}$ and $\Delta E^{\text {solu-solv }}$ are strongly correlated. The strong interaction between a charged group and a water cluster in the hydrated zwitterion weakens the water hydrogen bond networks, leading to large positive $\Delta E^{\text {solv }}$ values. The opposite tendency is found for the neutral systems. The total relative energies, $\Delta E^{\text {tot }}$ in the fifth column of Table 1 are negative for the water layers of thickness 4.0, 4.5 and 5.0 $\AA$. This means that there is no qualitative change in the relative neutral-zwitterion stabilities as the number of water molecules increases; the hydrated zwitterionic systems are always more stable. For the 3.0 and $3.5 \AA$ water layer systems, the relative energies are positive. Even though the zwitterionic system gains relative stability in water layers of 4.0, 4.5 and 5.0 $\AA$, the neutral system shows the relative stability at the 3.0 and $3.5 \AA$ water layers. The interaction between the $\mathrm{COO}^{-}$and $\mathrm{NH}^{3+}$ groups of zwitterionic trialanine and nearby water molecules strongly contributes to the stability of the zwitterion. According to the optimized geometries of zwitterionic trialanine with 3.0, 3.5, 4.0, 4.5 and $5.0 \AA$ water layers, $3,4,5,5,5$ water molecules directly interact with the $\mathrm{COO}^{-}$group and 2, 2, 3, 3, 3 water molecules interacting with the $\mathrm{NH}^{3+}$ group, respectively.

Table 1. Relative energy $\Delta E^{\text {tot }}$ contributions $(\mathrm{kJ} / \mathrm{mol})$ for FMO-RHF/EFP (solvent by $\mathrm{EFP})$ for hydrated zwitterionic trialanine relative to the neutral form

\begin{tabular}{ccccc}
\hline Water layer $(\AA)$ & $\Delta E^{\text {solu }}$ & $\Delta E^{\text {solv }}$ & $\Delta E^{\text {solu- solv }}$ & $\Delta E^{\text {tot }}$ \\
\hline $3.0(19)$ & 278.31 & 66.09 & -339.85 & 4.54 \\
$3.5(29)$ & 272.29 & 117.60 & -338.39 & 1.50 \\
$4.0(44)$ & 289.64 & 212.15 & -521.32 & -19.52 \\
$4.5(54)$ & 285.68 & 214.58 & -516.14 & -15.88 \\
$5.0(70)$ & 308.63 & 207.09 & -527.93 & -12.22 \\
\hline
\end{tabular}

The internal solute $\Delta E^{\text {solu }}$ and solvent $\Delta E^{\text {solv }}$ energies as well as the solute-solvent interaction $\Delta E^{\text {solu-solv }}$. The cc-pVDZ basis set is used. The number of water molecules is shown in parentheses

Tables 2 and 3 list the interaction energies $\Delta E^{\text {int }}$ of the $\mathrm{COO}^{-}$and $\mathrm{NH}_{3}{ }^{+}$groups with water molecules forming hydrogen bonds with these groups as a function of the incremental water layers and the corresponding optimized hydrogen bond lengths, respectively. The interaction energies are obtained as follows: the solute and the number of interacting water molecules forming hydrogen bonds directly with the carboxyl group are extracted from the fully solvated system. Then, the interaction energies between the solute and these water molecules are computed by infinitely separating the solute and this small water cluster. This is a very important test of the performance of the EFP method because it describes a very strong interaction between ionic $\left(\mathrm{COO}^{-}\right.$and $\left.\mathrm{NH}_{3}{ }^{+}\right)$groups and water molecules, which appears to be difficult for continuum models such as $\mathrm{PCM}^{45}$. 
Table 2. Interaction energy $\Delta E^{\text {int }}(\mathrm{kJ} / \mathrm{mol})$ between the zwitterionic trialanine and water molecules forming hydrogen bonds with the $\mathrm{COO}^{-}$and $\mathrm{NH}_{3}{ }^{+}$groups with the cc-pVDZ basis set (extracted from the large fully optimized structures with the thickness of water layers given in $\AA$ )

\begin{tabular}{|c|c|c|c|c|}
\hline \multirow[t]{2}{*}{ Water layer $(\AA)$} & \multirow{2}{*}{$E^{\text {solu-solv,zwit }}$} & \multicolumn{2}{|c|}{$\begin{array}{c}\text { No. of water molecules forming } \\
H \text {-bonds with }\end{array}$} & \multirow[t]{2}{*}{$\Delta E^{i n t}$} \\
\hline & & $\mathrm{COO}^{-}$group & $\mathrm{NH}_{3}{ }^{+}$group & \\
\hline 3.0 & -624.06 & 3 & 2 & -263.12 \\
\hline 3.5 & -740.43 & 4 & 2 & -298.61 \\
\hline 4.0 & -973.78 & 5 & 3 & -388.00 \\
\hline 4.5 & -1020.32 & 5 & 3 & -398.39 \\
\hline 5.0 & -998.68 & 5 & 3 & -394.03 \\
\hline
\end{tabular}

Table 3. Hydrogen bond lengths $(\AA)$ between the $\mathrm{COO}^{-}$and $\mathrm{NH}_{3}^{+}$groups of the hydrated zwitterionic trialanine and EFP water molecules (optimized with cc-pVDZ).

\begin{tabular}{ccccccccc}
\hline \multirow{2}{*}{$\begin{array}{c}\text { Water layer } \\
(\AA)\end{array}$} & \multicolumn{9}{c}{ Hydrogen bond lengths with } \\
\cline { 2 - 9 } $\mathrm{COO}^{-}$group & \multicolumn{5}{c}{$\mathrm{NH}_{3}{ }^{+}$group } \\
\hline $3.0(3,2)$ & 1.783 & 1.956 & 1.816 & - & - & 1.711 & 1.859 & - \\
$3.5(4,2)$ & 1.889 & 1.910 & 1.973 & 1.858 & - & 1.765 & 1.946 & - \\
$4.0(5,3)$ & 1.948 & 1.926 & 1.825 & 1.869 & 1.865 & 1.861 & 1.888 & 1.841 \\
$4.5(5,3)$ & 1.822 & 1.794 & 1.817 & 2.070 & 2.100 & 1.873 & 1.885 & 1.790 \\
$5.0(5,3)$ & 1.949 & 1.882 & 1.841 & 2.060 & 2.120 & 1.890 & 1.891 & 2.090 \\
\hline \multicolumn{4}{c}{ The numbers of water molecules are given in parentheses. }
\end{tabular}

It is interesting that the interaction energies $\Delta E^{\text {int }}$ between ionic groups of zwitterionic system and interacting water molecules shown in Table 2 reach nearly $40 \%$ of the total solute-solvent interaction energies $E^{\text {solu-solv,zwit }}$. It can be seen in Table 2 that more number of water molecules in the 4.0, 4.5 and 5.0 $\AA$ layer interact more strongly with the ionic group than do the nearest neighbor waters in the 3.0 , or $3.5 \AA$ layers. This may be because the larger clusters have eight direct solute-solvent hydrogen bonds, while the 3.0 and $3.5 \AA$ clusters have only five and six solute-solvent hydrogen bonds, respectively.

Table 4 displays the torsion angles of both neutral and zwitterionic systems in gas phase and in solvent phase (water layer of $5.0 \AA$ ). The deviation of $\varphi$ and $\psi$ angles are not appreciable in the hydrated neutral system, where as in the hydrated zwitterionic system $\varphi$ and $\psi$ angles are twisted by $20.26^{\circ}$ and $72^{\circ}$, respectively.

Table 4. Torsion angles (in degrees) of trialanine in gas phase and in aqueous medium (optimized with cc-pVDZ basis set)

\begin{tabular}{|c|c|c|c|c|}
\hline \multirow[b]{2}{*}{ Trialanine molecule in } & \multicolumn{2}{|c|}{ Neutral system } & \multicolumn{2}{|c|}{ Zwitterionic system } \\
\hline & $\begin{array}{c}\varphi \\
\text { (c3-n12- } \\
\text { c13-c14) }\end{array}$ & $\begin{array}{c}\psi \\
\text { (n12-c13- } \\
\text { c14-n22) }\end{array}$ & $\begin{array}{c}\varphi \\
\text { (c3-n13- } \\
\text { c14-c15) }\end{array}$ & $\begin{array}{c}\psi \\
\text { (n13-c14- } \\
\text { c15-n23) }\end{array}$ \\
\hline $\begin{array}{c}\text { solvent phase } \\
\text { (Water laver of } 5.0 \AA \text { ) }\end{array}$ & -154.33 & -165.52 & -164.33 & -149.69 \\
\hline gas phase & -155.19 & -160.69 & -156.61 & -169.95 \\
\hline
\end{tabular}




\section{Conclusion}

In this study the geometry optimization of hydrated neutral and zwitterionic trialanine in water layers of 3.0, 3.5, 4.0, 4.5 and $5.0 \AA$ is carried out via FMO-RHF/EFP model. The dihedral angles of both zwitterionic and neutral systems in gas phase and in solvent phase (water cluster of $5.0 \AA$ ) were studied. The hydrated zwitterionic trialanine is more stable than the neutral form. An interesting finding is that the stabilities do not increase monotonically with the water cluster size. In water layers of lower thickness (3.0 and $3.5 \AA$ ) number of water molecules may not sufficient to interact completely with the trialanine.

\section{References}

1. He X and Merz K M, J Chem Theory Comput., 2010, 6(2), 405.

2. Fedorov D G and Kitaura K, J Chem Phys., 2004, 120, 6832.

3. Fedorov D G and Kitaura K, The Fragment Molecular Orbital Method: Practical Applications to Large Molecular Systems (CRC Press, Boca Raton, FL, 2009).

4. Fedorov D G and Kitaura K, J Phys Chem A, 2007, 111, 6904.

5. Kitaura K, Sugiki S I, Nakano T, Komeiji Y and Uebayasi M, Chem Phys Lett., 2001, 336, 163-170.

6. Fedorov D G, Ishida T, Uebayasi M and Kitaura K, J Phys Chem A, 2007, 111, 2722-2732.

7. Nagata T, Fedorov D G and Kitaura K, Chem Phys Lett., 2009, 475, 124.

8. Komeiji Y, Mochizuki Y, Nakano T and Fedorov D G, $J$ Mol Struct THEOCHEM, 2009, 898(1-3), 2-7.

9. Fujita T, Watanabe H and Tanaka S, J Phys Soc Jpn., 2009, 78, 104723.

10. Fujiwara T, Mochizuki Y, Komeiji Y, Okiyama Y, Mori H, Nakano T and Miyoshi E, Chem Phys Lett., 2010, 490, 41-45.

11. Komeiji Y, Mochizuki Y and Nakano T, Chem Phys Lett., 2010, 484, 380-386.

12. Nagata T, Fedorov D G, Sawada T, Kitaura K and Gordon M S, J Chem Phys., 2011, 134(3), 034110.

13. Voet D and Voet J G, Biochemistry, $3^{\text {rd }}$ Ed., Wiley, New York, 2004.

14. Creighton T E, Proteins: Structure and Molecular Properties, $2^{\text {nd }}$ Ed., Freeman W H, New York, 1993.

15. Blanco S, Lessari A, Lopez J C and Alonso J L, J Am Chem Soc., 2004, 126, 11675-11683.

16. Csaszar A G, J Phys Chem., 1996, 100(9), 3541-3551.

17. Ling S, Yu W, Huang Z, Lin Z, Haranczyk M and Gutowski M, J Phys Chem A, 2006, 110, 12282.

18. Gutowski M, Skurski P and Simons J, J Am Chem Soc., 2000, 122, 10159-10162.

19. Radzicka A and Wolfenden R, Biochem., 1988, 27(5), 1664.

20. Wolfenden R, Andersson L, Cullis P M and Southgate C C B, Biochem., 1981, 20(4), 849-855.

21. Federov D G, Kitaura K, Li H, Jensen H J and Gordon M S, J Comput Chem., 2006, 27, 976-985.

22. Li H, Fedorov D G, Nagata T, Kitaura K, Jensen J H and Gordon M S, J Comput Chem., 2010, 31, 778.

23. Day N P, Jensen H J, Gordon M S and Webb P S, J Chem Phys., 1996, 105, 1968.

24. Gordon M S, Freitag M A, Bandyopadhyay P, Jensen J H, Kairys V and Stevens W J, J Phys Chem A, 2001, 105, 293-307. 
25. Nagata T, Fedorov D G, Kitaura K and Gordon M S, J Chem Phys., 2009, 131, 024101.

26. Dmitriy S C, Tateki Ishida and Levy R M, J Phys Chem B, 2004, 108, 19487-19495.

27. Ivan M D, Karl J J, Andrey A G and Risto M N, J Phys Chem B, 2007, 111, 4227.

28. Jensen J H and Gordon M S, J Am Chem Soc., 1995, 117, 8159-8170.

29. Aikens C M and Gordon M S, J Am Chem Soc., 2006, 128(39), 12835-12850.

30. Sicinska D, Paneth P and Truhlar D G, J Phys Chem B, 2002, 106, 2708.

31. Tajkhorshid E, Jalkanen K J and Suhai S, J Phys Chem B, 1998, 102, 5899.

32. Ellzy M W, Jensen J O, Hameka H F and Kay J G, J Spectrochim Acta A, 2003, 59, 2619-2633.

33. Frimand K, Bohr H, Jalkanen K J and Suhai S, J Chem Phys., 2000, 255, 165-194.

34. Han W G, Jalkanen K J, Elstner M and Suhai S, J Phys Chem B, 1998, 102, 2587-2602.

35. Poon C D, Samulski E T, Weise C F and Weisshaar J C, J Am Chem Soc., 2000, 122, 5642-5643.

36. Weise C F and Weisshaar J C, J Phys Chem B, 2003, 107, 3265-3277.

37. Yuguang M and Gerhard S, J Phys Chem B, 2002, 106, 5294-5301.

38. Wei D, Guo H and Salahub D R, Phys Rev E, 2001, 64, 11907.

39. Leung K and Rempe S B, J Chem Phys., 2005, 122, 184506.

40. Hanwell M D, Curtis D E, Lonie D C, Vandermeersch T, Zurek E and Hutchison G R, J Cheminformatics, 2012, 4(1), 17.

41. Pedretti A, Villa L and Vistoli G, J Mol Graphics Model., 2002, 21, 47-49.

42. Dunning T H, J Chem Phys., 1989, 90, 1007.

43. Schmidt M W, Baldridge K K, Boatz J A, Elbert S T, Gordon M S, Jensen J H, Koseki S, Matsunaga N, Nguyen K A, Su S J, Windus T L, Dupuis M and Montgomery J A, J Computational Chem., 1993, 14, 1347.

44. Advances in electronic structure theory GAMESS a decade later Gordon M S, Schmidt M W in Theory and Applications of Computational Chemistry, the first forty years Dykstra C E, Frenking G, Kim K S, Scuseria G E, editors (Elsevier, Amsterdam). 2005, Chapter 41, pp1167.

45. Mullin J M and Gordon M S, J Phys Chem B, 2009, 113, 8657-8669. 